\title{
Discovery of a magnetic neutron star in X-ray transient IGR J01583+6713
}

\author{
W. Wang \\ National Astronomical Observatories, Chinese Academy of Sciences, Beijing 100012, PR China \\ e-mail: wangwei@bao.ac.cn
}

Received 28 August 2009 / Accepted 5 March 2010

ABSTRACT

\begin{abstract}
Aims. IGR J01583+6713 is a new transient source discovered by the INTEGRAL/IBIS hard X-ray surveys. Optical observations have provided evidence that it is a high mass X-ray binary, but its nature remains unclear. Methods. We used the INTEGRAL/IBIS data to study IGR J01583+6713 during its outburst.

Results. We present the temporal profiles and spectral properties of IGR J01583+6713 around its outburst on 2005 Dec. 6. During the outburst, the mean X-ray luminosity reached around $4 \times 10^{35} \mathrm{erg} \mathrm{s}^{-1}$ in the energy range of 20-100 keV. The continuum spectrum can be fitted by a bremsstrahlung model of $k T \sim 35 \mathrm{keV}$ or a power-law model of $\Gamma \sim 2.1$. In addition, the electron resonant cyclotron absorption lines were detected at $\sim 35 \mathrm{keV}$ and possible at $\sim 67 \mathrm{keV}$, implying that a magnetic neutron star of $B \sim 4 \times 10^{12} \mathrm{G}$ is located in IGR J01583+6713. We class IGR J01583+6713 as a transient X-ray sources with a magnetic neutron star. After the outburst, the flux of IGR J01583+6713 decreased and could not be detected by IBIS after 2005 Dec. 10. During the outburst, we cannot confirm the possible pulse period at $469 \mathrm{~s}$ previously reported and did not detect the modulation signals in the range of 200-2000 s with the IBIS observations.

Conclusions. The transient hard X-ray source IGR J01583+6713 contains a magnetic neutron star, which would help to understand its transient nature.
\end{abstract}

Key words. stars: individual: IGR J01583+6713 - stars: neutron - binaries: general - X-rays: binaries

\section{Introduction}

The transient X-ray source IGR J01583+6713 was discovered by the the soft Gamma-ray Imager (IBIS/ISGRI) onboard The INTErnational Gamma-Ray Astrophysics Laboratory (INTEGRAL) during the observation of Cas A region on 2005 December 6 (Steiner et al. 2005). Subsequent INTEGRAL observations in the same region from 2005 Dec. 8-10 showed that the X-ray flux of IGR J01583+6713 was decreasing (Steiner et al. 2005). Swift observations also found this source on 2005 Dec. 13, and revealed that it is highly absorbed with $N_{\mathrm{H}} \sim$ $10^{23} \mathrm{~cm}^{-2}$ (Kennea et al. 2005). Follow-up optical and infrared observations identified the X-ray source as a Be star (Halpern et al. 2005). Based on optical spectroscopy, Masetti et al. (2006) classified the counterpart as an early-type star (O8 III or O9 V) in the Galaxy with a distance of $\sim 6.4 \mathrm{kpc}$, ruling out the possibility of both a supergiant companion and the source either being a low-mass X-ray binary or a cataclysmic variable.

Kaur et al. (2008) carried out a multiwavelength study on the transient source IGR J01583+6713, identified the spectral type of the companion star to be B2 IVe, and suggested a distance of $\sim 4 \mathrm{kpc}$. From the Swift observations, they also reported a possible pulse period of $\sim 469 \mathrm{~s}$, but the evidence of pulsation was only marginal (Kaur et al. 2008), requiring additional confirmation.

We present results of the INTEGRAL/IBIS observations during the outburst state of IGR J01583+6713 that occurred in 2005 December. By reanalyzing these data, we attempt aim to study the hard X-ray spectral properties of the source both during and after the outburst. During the outburst state, we attempt search for the possible pulsation period using IBIS data. In Sect. 2, observations and data analysis are presented. We show the spectral results in Sect. 3 and timing analysis in Sect. 4. A summary of our results and brief discussions is presented in the last section.

\section{INTEGRAL/IBIS observations and data analysis}

The present database was obtained from the INTEGRAL observations of the Cas A region performed from 2005 December 5-16 (MJD 53 709-53 720), from the part of INTEGRAL satellite revolutions 384-387. We used data from the coded mask imager IBIS/ISGRI (Lebrun et al. 2003; Ubertini et al. 2003) for a total exposure of $\sim 450 \mathrm{ks}$ (also see Table 1) after screening for solar-flare events and erratic count fluctuations due to passages through the Earth's radiation belts.

The analysis was performed with the standard INTEGRAL off-line scientific analysis (OSA, Goldwurn et al. 2003) software, ver. 7.0. Individual pointings processed with OSA 7.0 were mosaicked to create sky images according to the methods and processes described in Bird et al. (2007). And we used the 20-60 keV band for source detection and to quote fluxes (see Fig. 1 and Table 1).

In revolution 384 (2005 Dec. 5-7), X-ray source IGR J01583+6713 was detected at the outburst state with a significance level of $\sim 16.2 \sigma$. And from Dec. $8-10$, the mean flux of IGR J01583+6713 decreased to less than half of its value in its outburst state, with a detection significance level of $\sim 7.3 \sigma$. But after 2005 Dec. 11, IGR J01583+6713 could not be detected by IBIS (Table 1). In the present analysis, we use the data in two revolutions 384 and 385 for the scientific studies. 
Table 1. INTEGRAL/IBIS observations of the field around IGR J01583+6713.

\begin{tabular}{lcccl}
\hline \hline Rev. Num. & Date & On-source time $(\mathrm{ks})$ & Mean count rate s $^{-1}$ & Detection level \\
\hline 384 & 2005 Dec. 05-07 & 118 & $2.91 \pm 0.15$ & $16.2 \sigma$ \\
385 & 2005 Dec. 08-10 & 132 & $1.4 \pm 0.2$ & $7.3 \sigma$ \\
386 & 2005 Dec. 11-13 & 121 & $<0.8(2 \sigma)$ & $<4 \sigma$ \\
387 & 2005 Dec. 14-16 & 98 & $<0.9(2 \sigma)$ & $<4 \sigma$ \\
\hline
\end{tabular}

Notes. The time intervals of observations in the revolution number and the corresponding dates, the corrected on-source exposure time are listed. And mean count rate and the detection significance level value in the energy range of 20-60 keV were also shown.

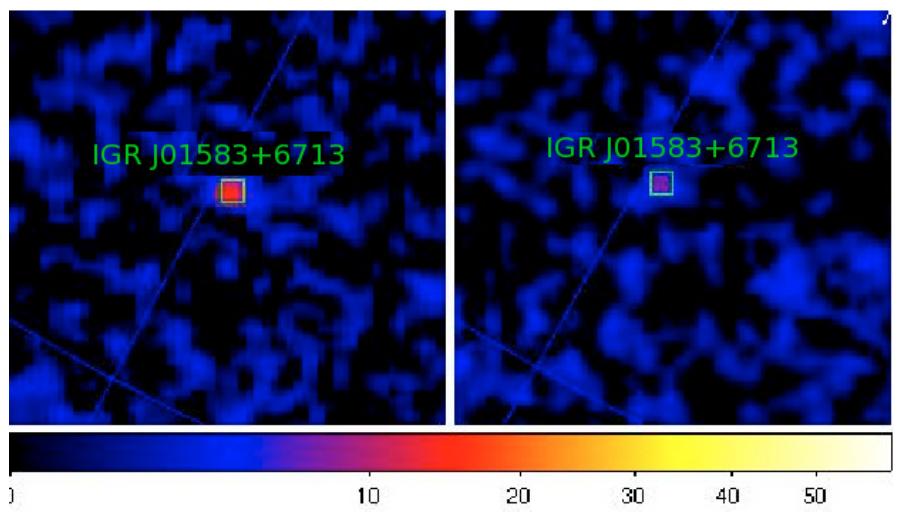

Fig. 1. Significance mosaic maps around IGR J01583+6713 in Equatorial J2000 coordinates as seen with INTEGRAL/IBIS in the energy range of 20-60 keV in two revolutions: 384 (left, outburst), 385 (right, flux decreasing). False color representation of significance is displayed on a logarithmic scale.

In the IBIS observations of IGR J01583+6713, it should also be noted that some observations had large off-axis angles (e.g., $>12^{\circ}$ ). Because the mask transmission at large off-axis angles is affected by the mask support structure in a complex way that cannot not be easily modelled, timing analysis for large off-axis angles $>12^{\circ}$ could be subject to significant systematic errors due to limitations in the off-axis corrections performed (private communications from the IBIS group). The flux error bars may also become significantly larger for large off-axis angles due to lower effective exposures. Therefore, in the scientific studies (spectral and timing analysis) of IGR J01583+6713, we only use the data with the off-axis angles $<12^{\circ}$ in the following analysis. The remaining on-source time in the following science analysis is then about $51 \mathrm{ks}$ and $75 \mathrm{ks}$ for Rev 384 and Rev 385, respectively.

Extraction of spectra and light curves for then two revolutions 384 and 385 was also carried out in the OSA software system. In the timing analysis, because of the sensitivity limits of IBIS/ISGRI detector, the minimum time intervals are taken to be about $70 \mathrm{~s}$ and $\sim 300 \mathrm{~s}$ for the outburst state Rev 384 and Rev 385 , respectively.

\section{Spectral properties}

As a transient X-ray source, IGR J01583+6713 was only detected by IBIS during the outburst state around 2005 Dec. 6 and just few days after the outburst. In this section, we present the spectral properties of the transient source IGR J01583+6713 in the range 20-200 keV during the outburst (Rev 384) and after the outburst (Rev 385) separately. The spectral analysis software package used is XSPEC 12.4.0x (Arnaud 1996).

\footnotetext{
${ }^{1}$ http://heasarc.gsfc.nasa.gov/docs/xanadu/xspec/
}
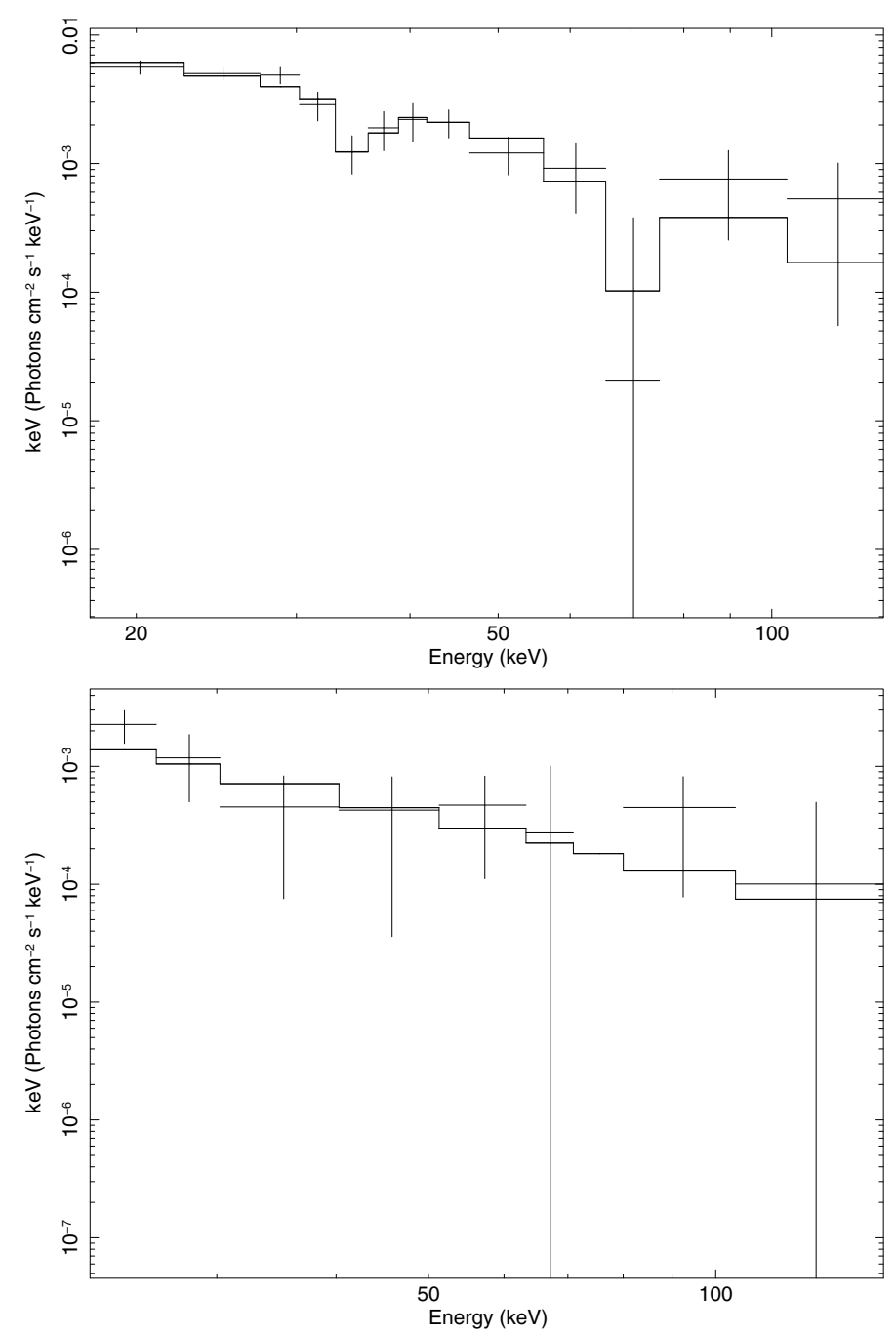

Fig. 2. Top: the hard X-ray unfolded spectrum of IGR J01583+6713 during the outburst from 2005 Dec. 6-7. The spectrum was fitted using either a bremsstrahlung model of $k T \sim 35.2 \pm 4.1$ plus two cyclotron resonant absorption lines at $\sim 35.3 \pm 1.6 \mathrm{keV}$ and $\sim 67.9 \pm 4.8 \mathrm{keV}$, or a single power-law model plus two lines at $35.1 \pm 2.1 \mathrm{keV}$ and $65.8 \pm 4.9 \mathrm{keV}$. Bottom: the hard X-ray unfolded spectrum of IGR J01583+6713 after the outburst from 2005 Dec. 8-10. The spectrum can be fitted by a single power law model of $\Gamma \sim 2.7 \pm 0.6$. See details in the text.

In revolution 384, IGR J01583+6713 was detected at a high significance level $(>16 \sigma)$. The spectrum of this source in the outburst state is presented in Fig. 2, top panel. We first used two models to fit the spectrum: a thermal bremsstrahlung model and a power-law model. The $20-120 \mathrm{keV}$ spectrum was fitted by a thermal bremsstrahlung model with $k T \sim 25.1 \pm 1.9 \mathrm{keV}$ (reduced $\chi^{2} \sim 1.211,11$ degree of freedom, thereafter d.o.f.). The 
Table 2. Different fits to the spectrum of IGR J01583+6713 during the outburst state.

\begin{tabular}{lccccc}
\hline \hline Model & $\Gamma / k T$ & $E_{\mathrm{cyc}, 0}(\mathrm{keV})$ & $E_{\mathrm{cyc}, 1}(\mathrm{keV})$ & Flux $\left(10^{-10} \mathrm{erg} \mathrm{cm}^{-2} \mathrm{~s}^{-1}\right)$ & reduced $\chi^{2}$ \\
\hline power+cyclabs & $2.13 \pm 0.53$ & $35.1 \pm 2.1$ & $65.8 \pm 4.9$ & $1.99 \pm 0.42$ & $0.989(5$ d.o.f. $)$ \\
bremss+cyclabs & $35.2 \pm 4.1 \mathrm{keV}$ & $35.3 \pm 1.6$ & $67.9 \pm 4.8$ & $1.98 \pm 0.41$ & $0.828(5$ d.o.f. $)$ \\
\hline
\end{tabular}

Notes. Power implies a power law model, bremss implies a thermal bremsstrahlung model, cyclabs implies a resonant cyclotron absorption model. The hard X-ray fluxes in the range of $20-100 \mathrm{keV}$ in different fits are also given.

derived hard X-ray flux from $20-100 \mathrm{keV}$ was determined to be $(1.91 \pm 0.36) \times 10^{-10} \mathrm{erg} \mathrm{cm}^{-2} \mathrm{~s}^{-1}$, corresponding to a hard $\mathrm{X}$-ray luminosity of $\sim(3.8 \pm 0.7) \times 10^{35} \mathrm{erg} \mathrm{s}^{-1}$ when assuming a distance of $4 \mathrm{kpc}$ (Kaur et al. 2008). This spectrum was also fitted by a power-law model, with $\Gamma \sim 2.35 \pm 0.18$ (reduced $\chi^{2} \sim 1.290,11$ d.o.f.), and the derived flux $(20-100 \mathrm{keV})$ is about $(2.2 \pm 0.5) \times 10^{-10} \mathrm{erg} \mathrm{cm}^{-2} \mathrm{~s}^{-1}$, corresponding to a hard $\mathrm{X}$-ray luminosity of $\sim(4.4 \pm 0.9) \times 10^{35} \mathrm{erg} \mathrm{s}^{-1}$.

In both fits, we found that an absorption feature around 30-40 keV cannot be fitted, and another possible feature around $60-70 \mathrm{keV}$ also exists. These features are probably related to the electron cyclotron resonance scattering feature (CRSF) in the transient source. We therefore used the thermal bremsstrahlung/power-law model to fit the continuum, and added the cyclotron resonant absorption model by using the XSPEC cyclabs to the continuum fit (see Fig. 2 top panel). In the power-law fit case, we derived $\Gamma \sim 2.13 \pm 0.53$ in addition to a CRSF at $E_{\text {cyc }} \sim 35.1 \pm 2.1 \mathrm{keV}\left(\right.$ F-test probability: $3.9 \times 10^{-9}$ ) with a line width of $F W H M \sim 1.1 \pm 0.4 \mathrm{keV}$ and a depth $1.75 \pm 0.59$, and another feature at $65.8 \pm 4.9 \mathrm{keV}$ (F-test probability: 0.009) with a line width of $F W H M \sim 4.1 \pm 2.4 \mathrm{keV}$ and a depth $0.81 \pm 0.56$ (reduced $\chi^{2} \sim 0.989,5$ d.o.f.). We also carried out the fit with a bremsstrahlung model plus the CRSF on the spectrum. We then also found two absorption features in the fit, and obtained $k T \sim 35.2 \pm 4.1 \mathrm{keV}$ plus a CRSF at $35.3 \pm 1.6 \mathrm{keV}$ (F-test probability: $5.6 \times 10^{-10}$ ) with a $F W H M \sim 1.2 \pm 0.4 \mathrm{keV}$ and a depth of $\sim 1.98 \pm 0.71$, the other CRSF at $67.9 \pm 4.8 \mathrm{keV}$ (F-test probability: 0.011) with a $F W H M \sim 4.4 \pm 2.5 \mathrm{keV}$ and a depth of $\sim 0.93 \pm 0.55$ (reduced $\chi^{2} \sim 0.828,5$ d.o.f., see Fig. 2 , top panel and Table 2).

After the outburst from 2005 Dec. 8-10, IGR J01583+6713 was detected by IBIS at a lower significance level $\sim 7 \sigma$. The spectrum after the outburst is displayed in the bottom panel of Fig. 2. The $20-100 \mathrm{keV}$ spectrum could be described by a single power-law model of the photon index $\Gamma \sim 2.7 \pm 0.6$ (reduced $\chi^{2} \sim 0.929,7$ d.o.f.). No significant absorption features are detected. The derived flux is $\sim(7.1 \pm 2.8) \times 10^{-11} \mathrm{erg} \mathrm{cm}^{-2} \mathrm{~s}^{-1}$ from $20-100 \mathrm{keV}$, corresponding to a mean luminosity of $\sim(1.4 \pm 0.5) \times 10^{35} \mathrm{erg} \mathrm{s}^{-1}$.

\section{Timing analysis}

From one SWIFT observation, during which the X-ray intensity was higher, Kaur et al. (2008) had a possible pulse detection with a period of $469.2 \mathrm{~s}$. This detection was, however marginal. Thus, using the data of the IBIS observations during the outburst state of the transient hard X-ray source IGR J01583+6713, we attempt to search for the possible pulse period.

The light curve of IGR J01583+6713 from 2005 Dec. 6-7 observed by IBIS/ISGRI in the energy range of $20-60 \mathrm{keV}$ is shown in Fig. 3 top panel. The background count rate was subtracted and the barycentric corrections were also performed. The count rates were rebinned in intervals of $1000 \mathrm{~s}$ in the plot for clarity. During the outburst on 2005 Dec. 6, the count rate
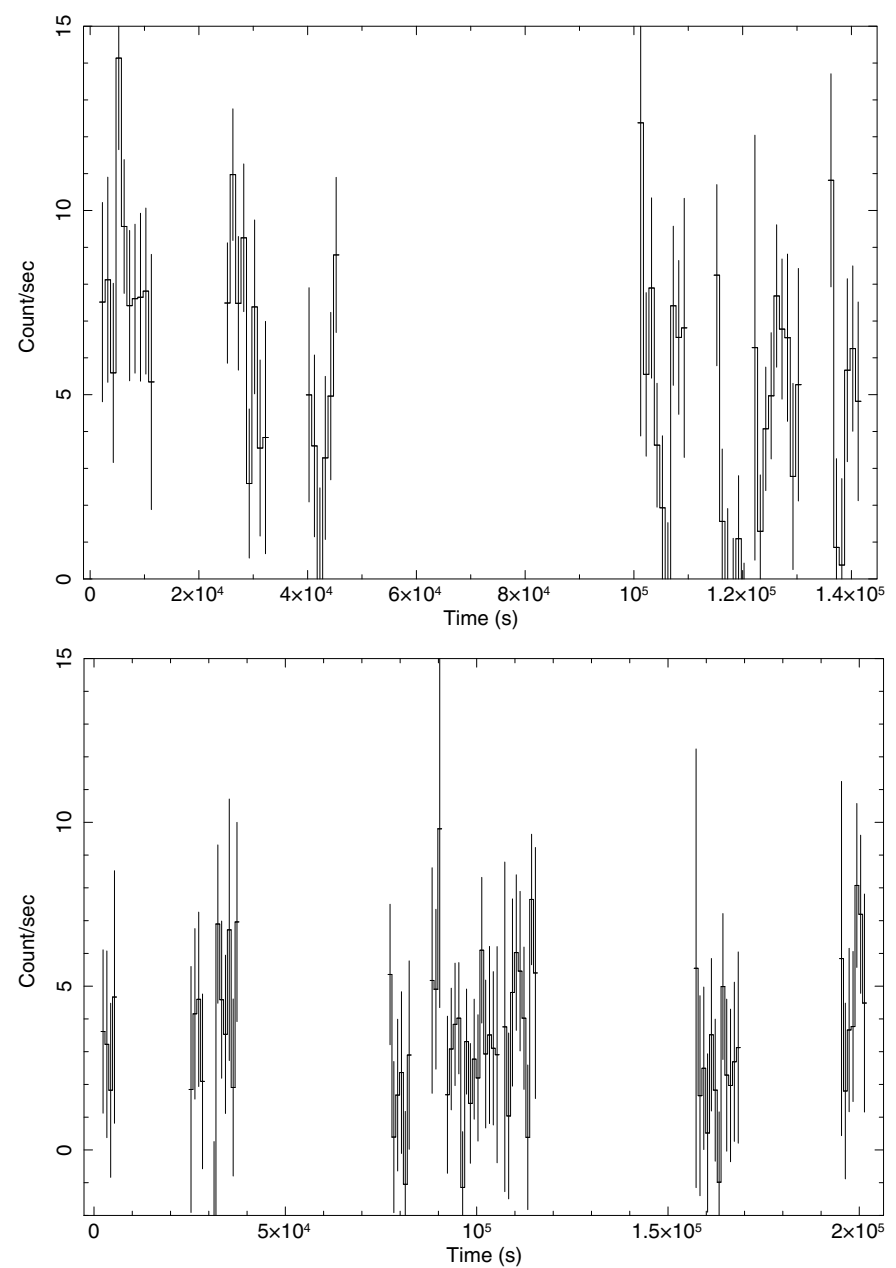

Fig. 3. The IBIS/ISGRI background subtracted light curve of IGR J01583+6713 from 20-60 keV. Top: the light curve in Rev 384 starting at MJD 53710.101 (2005 Dec. 6) with the bin time of 1000 s. Bottom: the light curve in Rev 385 starting at MJD 53712.159 (2005 Dec. 8) with the bin time of $1000 \mathrm{~s}$.

reached $\sim 10 \mathrm{cts} \mathrm{s}^{-1}$. On Dec. 7 , the rate decreased to $\sim 5 \mathrm{cts} \mathrm{s}^{-1}$. From 2005 Dec. $8-10$ (revolution 385, Fig. 3 bottom panel), the count rate was around $3 \mathrm{cts} \mathrm{s}^{-1}$. After the outburst on Dec. 6 , the X-ray flux of the transient source IGR J01583+6713 decreased.

To search for the modulation signal in the X-ray light curves, we applied the FFT to the IBIS/ISGRI light curves of IGR J01583+6713 during the outburst on 2005 Dec. 6. We used powspec of the Xronos software package ver. $5.21^{2}$ available from NASA's HEASARC for the power density spectrum analysis. To estimate the significance level of the possible detected peak signals in the power spectra, we considered the white and red noise significance levels. The $99 \%$ white noise significance level was estimated using Monte Carlo simulations (see

${ }^{2}$ http://heasarc.gsfc.nasa.gov/docs/xanadu/xronos/ 

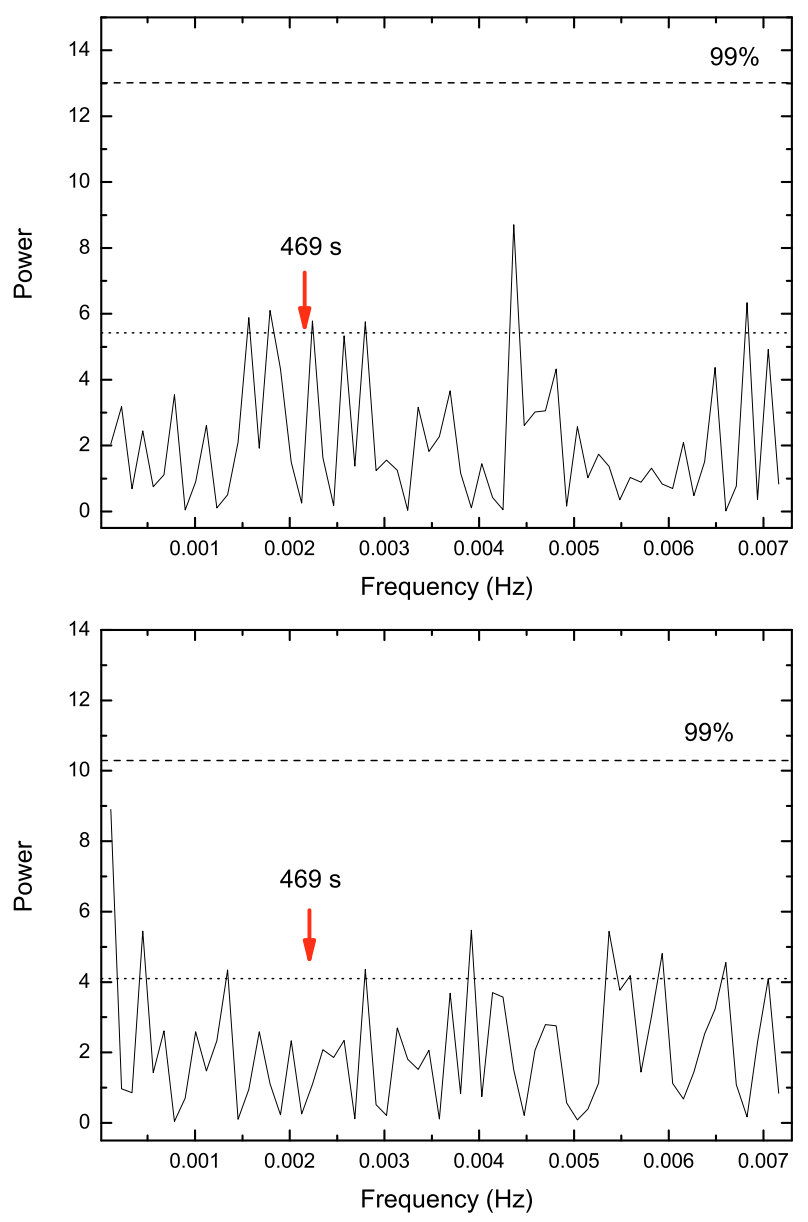

Fig. 4. The power spectra of the IBIS light curve of IGR J01583+6713 for the outburst state on 2005 Dec. 6: (top) from MJD 53710.10153 710.219; (bottom) MJD 53 710.370-53 710.457. The possible period at $469 \mathrm{~s}$ reported by Kaur et al. (2008) is also noted in both power spectra. The dashed and dotted lines present the 99\% significant levels for white noise and red noise respectively.

examples in Kong et al. 1998). The 99\% red noise significance level was estimated using the REDFIT35 subroutine (Schulz \& Mudelsee 2002) ${ }^{3}$, which fits a first-order autoregressive process to the time series to estimate the red-noise spectrum.

The hard X-ray count rates of IGR J01583+6713 in the outburst were obtained in several time intervals (see Fig. 3 top panel). In two time intervals, MJD 53710.101-53710.219 and MJD 53710.370-53710.457, the detected flux from IGR $\mathrm{J} 01583+6713$ had a count rate higher than $5 \mathrm{cts} \mathrm{s}^{-1}$, so we applied the FFT to these two light curves to search for possible modulation features. The derived power spectra of two outburst light curves were presented in Fig. 4, binned in $70 \mathrm{~s}$ intervals. The position of the pulse period at $469 \mathrm{~s}$ was identified in two power spectra, but no significant peak signals were found around $469 \mathrm{~s}$ in both power spectra. For the time interval MJD 53 710.101-53710.219, we found a possible peak feature at $\sim 230 \mathrm{~s}$ (Fig. 4 top panel), but it may be caused by the noise (significantly lower than the $99 \%$ white noise level and just a little higher than the $99 \%$ red noise level). In addition, this possible feature around $230 \mathrm{~s}$ was not found in the time interval MJD 53 710.370-53710.457. Therefore, using the power spectrum analysis of our present IBIS data, we did not detect

\footnotetext{
3 ftp://ftp.rz.uni-kiel.de/pub/sfb313/mschulz/ redfit35.zip
}
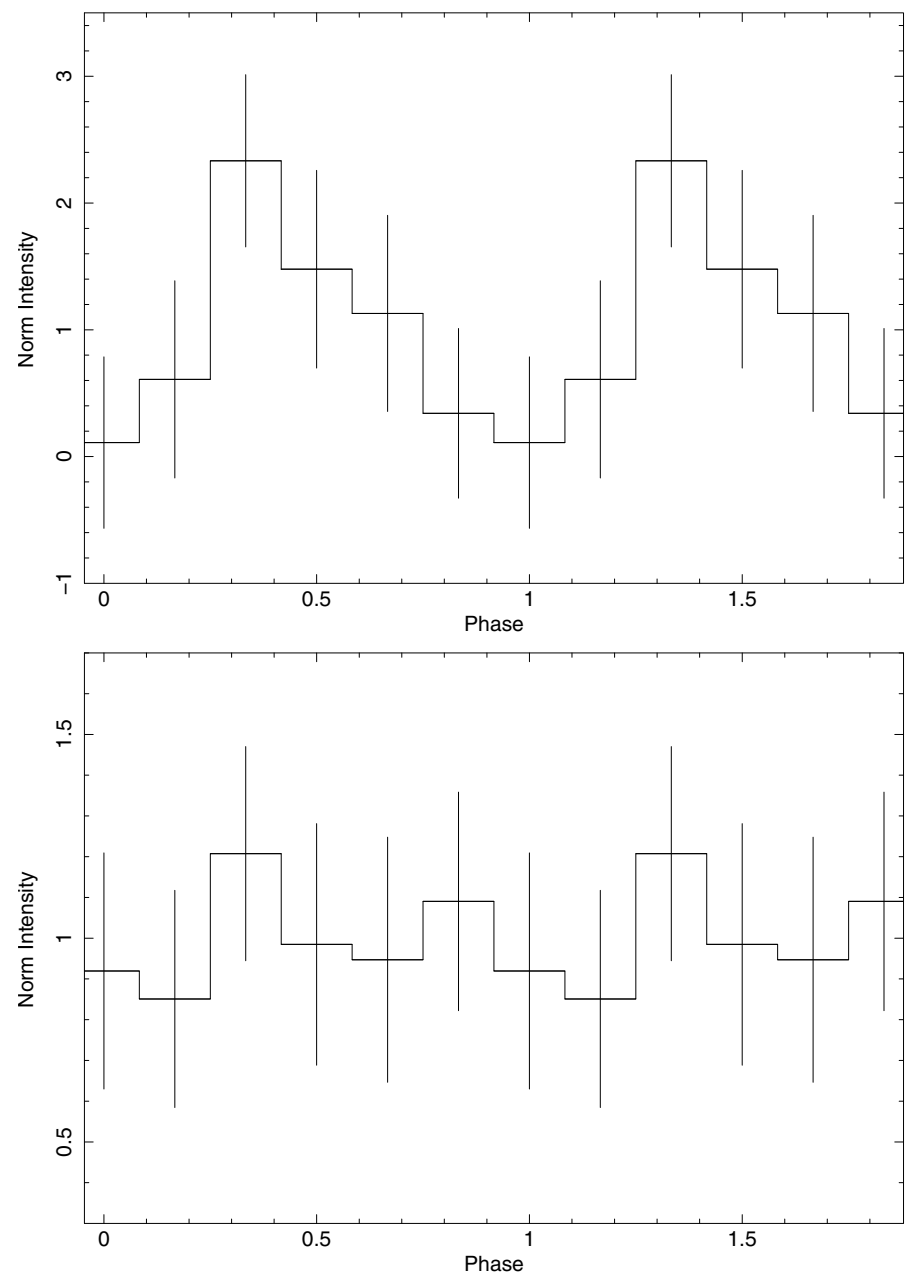

Fig. 5. The IBIS/ISGRI light curves (20-60 keV) of IGR J01583+6713 folded at a possible pulsation period (469 s) for the observational data of two time intervals in outbursts: MJD 53710.101-53 710.219 (top); and MJD 53 710.370-53 710.457 (bottom). The pulse profile is repeated once for clarity.

any possible modulation period signals in the X-ray light curves from 200-2000 s.

We note that the $469 \mathrm{~s}$ period was found by means of the pulse-folding technique (Kaur et al. 2008). We were also able to derive the folded profiles by folding the hard X-ray light curves at the possible pulse period. We folded the X-ray light curves using the efold tool of the Xronos software package. The folded pulse profiles were derived for two possible period values indicated in this work $469 \mathrm{~s}$ and $230 \mathrm{~s}$ (see Figs. 5 and 6). The folded profiles at both two periods of $469 \mathrm{~s}$ and $230 \mathrm{~s}$ from MJD 53710.101-53710.219 show the possible pulse features with the pulse fraction near $100 \%$, if we assume this pulse period to be true. From MJD 53710.370-53710.457, significant pulse features do not appear in the folded profiles (large error bars can make the profiles look almost flat). When only considering pulse-folded profiles, we may find two possible period values: $469 \mathrm{~s}$ and $230 \mathrm{~s}$. Only one of them may be the true period or both of them are not. However, both two period signals have not been detected in power spectrum analysis. Therefore the pulsefolding technique would not be a good tool for blindly searching for the possible period signal at the first step. The possible pulse period of $469 \mathrm{~s}$ cannot be confirmed with the IBIS data.

In addition, we could also check the possible pulse period at $469 \mathrm{~s}$ in a different way. In Fig. 7, we show the $P_{\text {spin }}-P_{\text {orbit }}$ 

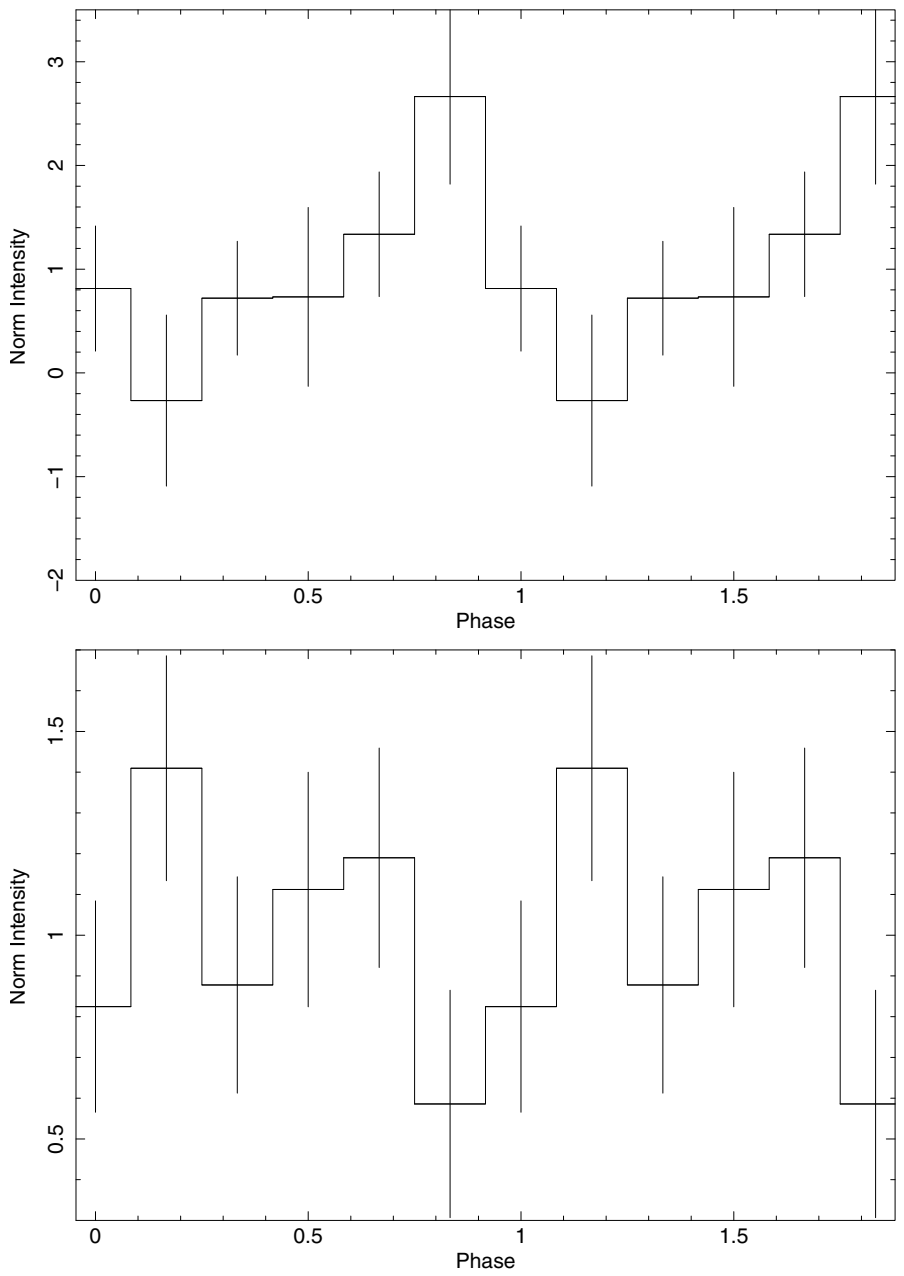

Fig. 6. The IBIS/ISGRI light curves (20-60 keV) of IGR J01583+6713 folded at a possible pulsation period $(230 \mathrm{~s})$ for the observational data of two time intervals in outbursts: MJD 53710.101-53 710.219 (top); and MJD $53710.370-53710.457$ (bottom). The pulse profile is repeated once for clarity.

diagram for known neutron star X-ray binaries (Corbet diagram, Corbet 1986). From optical identification (Masetti et al. 2006), IGR J01583+6713 may be a Be/X-ray star that might follow the previously found correlation between orbital period and pulse period in the classic Be star/neutron star binary systems (Corbet 1986). With the possible pulse period at 469 s, Kaur et al. (2008) estimated that the X-ray binary IGR J01583+6713 has an orbital period in the range 216-561 days assuming the maximum eccentricity of the orbit to be 0.47 for Be binaries as observed by Bildsten et al. (1997). Some neutron-star binary systems like underfilled Roche lobe supergiants and 4U 2206+54, 2S 0114+65 are powered by direct wind accretion. These systems may follow a $P_{\text {spin }}-P_{\text {orbit }}$ relation that is quite different from that of the Be transient systems. If IGR J01583+6713 were to follow the possible relation (i.e., one that is not clearly evident) of wind accretion systems, assuming a pulse period of 469 s, IGR J01583+6713 may have a possible orbital period in the range 3-12 days.

To search for the possible orbital modulation signal of IGR J01583+6713, we used the archival one-day average RXTE/ASM data ${ }^{4}$. We downloaded the one-day average RXTE/ASM data for IGR J01583+6713 from 2000 Jan to 2009 Dec. and applied the FFT to the ten years of light curve data.

${ }^{4}$ ASCII files available from http://xte.mit.edu/

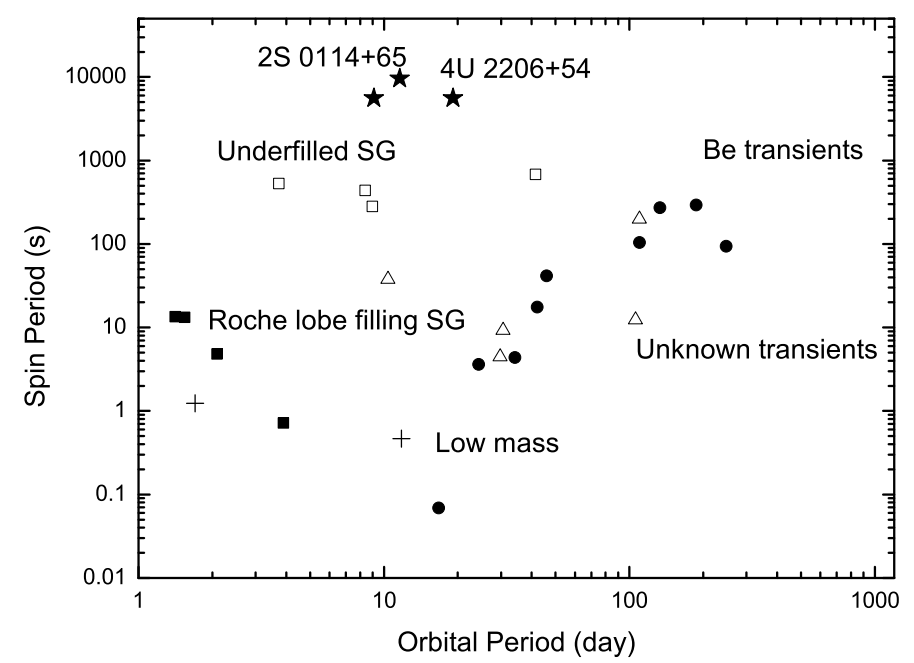

Fig. 7. The spin period-orbital period diagram for the accreting neutron star systems (the Corbet diagram, Corbet 1986). The data point for $2 \mathrm{~S} 0114+65$ is taken from Farrell et al. (2008); the data point for $4 \mathrm{U} 2206+54$ is taken from Wang (2009), two possible values of orbital period: 9.6 day and 19.1 day are both shown in this diagram; other points are taken from Bildsten et al. (1997). There exist a positive correlation between $P_{\text {spin }}-P_{\text {orbit }}$ for the Be transient systems; and a possible negative correlation for the disk-accretion system including Roche lobe filling supergiants and low mass systems. For the underfilled Roche lobe supergiants and two long-pulsation systems $2 \mathrm{~S} 0014+65$ and $4 \mathrm{U}$ $2206+54$, which all belong to wind-fed accretion systems, the possible relation $P_{\text {spin }}-P_{\text {orbit }}$ differing from that of the Be transients.

The power spectrum of the RXTE/ASM data is presented in Fig. 8, but no significant features are apparent. In the range of 216-561 days, no peak features were found, and in the range of 3-12 days, two possible signals at $\sim 7.7$ days and $\sim 10.6$ days could be noise signals which are significantly lower than the 99\% white noise level. Therefore, with the RXTE/ASM data, we have not found any possible orbital period within the interval 3-1000 days, so we also cannot confirm the possible pulsed period at 469 s yet assuming the possible correlation between the spin period and orbital period in neutron star binaries.

\section{Summary and discussion}

We have attempted to determine the nature of the transient hard X-ray source IGR J01583+6713 using IBIS/ISGRI observations acquired during the outburst state around 2005 Dec. 6. During the outburst, IGR J01583+6713 had an average luminosity of $\sim 4 \times 10^{35} \mathrm{erg} \mathrm{s}^{-1}$ in the energy interval $20-100 \mathrm{keV}$. The spectrum of IGR J01583+6713 can be described by both a thermal bremsstrahlung model with $k T \sim 35 \mathrm{keV}$ plus two resonant cyclotron absorption lines at $\sim 35.3 \pm 1.6 \mathrm{keV}$ and $\sim 67.9 \pm 4.8 \mathrm{keV}$ and a single power-law model plus two lines at $35.1 \pm 2.1 \mathrm{keV}$ and $65.8 \pm 4.9 \mathrm{keV}$. We can estimate the magnetic field of the compact object in IGR J01583+6713 according to a formula

$\left[B / 10^{12} \mathrm{G}\right]=\left[E_{\mathrm{cycl}} / 11.6 \mathrm{keV}\right](1+z)$,

where $E_{\text {cycl }}$ is the energy of the fundamental line, which we assume to be $E_{\text {cycl }}=35 \pm 3 \mathrm{keV}$, and $z$ is the gravitational redshift near the surface of the neutron star. For a canonical neutron star of $1.4 M_{\odot}$ with a radius of $10 \mathrm{~km}$, we can take $z \sim 0.3$ (Kreykenbohm et al. 2004). Then we found a magnetic field of $\sim(4.0 \pm 0.4) \times 10^{12} \mathrm{G}$ for IGR J01583+6713. Thus, the compact object in IGR J01583+6713 should be a magnetic neutron star. 


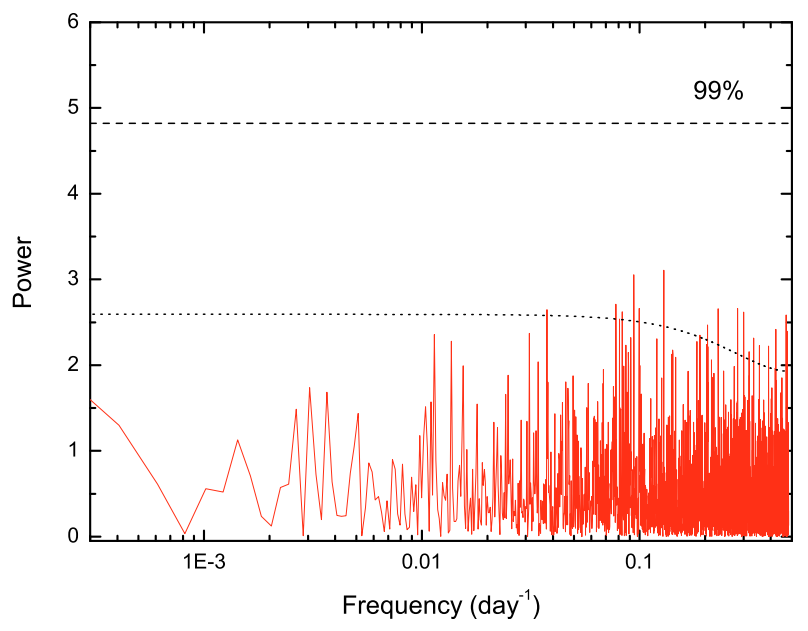

Fig. 8. The power spectrum of the ASM light curve of IGR J01583+6713 from 2000 Jan. to 2009 Dec. We have not detected any significant modulation signals during the interval 4-1000 days. The possible peak features at $\sim 7.7$ days and $\sim 10.6$ days are probably noise signals, which are significantly lower than the $99 \%$ white noise level (dashed line). The dotted line presents the $99 \%$ red noise significant level.

Timing analysis was also carried out during the outburst to search for the possible pulsation period. The possible pulsation period at $\sim 469$ s reported by Kaur et al. (2008) cannot be confirmed by our power spectrum analysis. In the time interval of MJD 53710.101-53710.219, a possible feature at $230 \mathrm{~s}$ was found in the power spectrum, which is still significantly lower than the $99 \%$ white noise level. However, the folded light curves at both $230 \mathrm{~s}$ and 469.2 s from MJD 53710.101-53710.219 show possible pulse profiles. The pulse-folding technique may therefore provide some incorrect information about the blind period search. In addition, with the present IBIS observations, we have not detected any possible modulation period in the range 200-2000 s, and present RTXE/ASM data does no exhibit any orbital modulation signals in the range 3-1000 days.

After the outburst, the hard X-ray flux of IGR J01583+6713 decreased according to our observations. During the interval 2005 Dec. $8-10$, the mean X-ray luminosity was around $\sim 1.4 \times$ $10^{35} \mathrm{erg} \mathrm{s}^{-1}$ in the range of $20-100 \mathrm{keV}$. The spectrum could be fitted by a power-law model of $\Gamma \sim 2.7$. Because of the detection of a low significance level $(\sim 7 \sigma)$, the CRSF was not found after the outburst. The derived power-law slope of $\sim 2.7$ is generally steeper than those of other neutron star X-ray binaries (around 1.7-2.1, e.g. see Wang 2009; Farrell et al. 2008). A large uncertainty of 0.6 still exists, which requires confirmation.

The transient X-ray pulsar IGR J01583+6713 may represent a link to the supergiant fast X-ray transients (SFXTs) in some supergiant high mass X-ray binaries (e.g., Sguera et al. 2006) belonging to wind-fed systems. These transients show soft gamma-ray time-structured bursts of durations from several hours to about 2 days detected by INTEGRAL/IBIS observations. The mean X-ray luminosity reaches around $10^{35}-10^{36} \mathrm{erg} \mathrm{s}^{-1}$, while in quiescence, the X-ray luminosity is only $\sim 10^{33} \mathrm{erg} \mathrm{s}^{-1}$ (Sguera et al. 2006). These behaviors of SFXTs are similar to those of the transient X-ray pulsar IGR J01583+6713, though IGR J01583+6713 has a different companion from SFXTs. Soft X-ray monitoring of IGR J01583+6713 found that in quiescence after the outburst, the X-ray luminosity of IGR J01583+6713 decreased to several $10^{33} \mathrm{erg} \mathrm{s}^{-1}$ (Kaur et al. 2008). Both the origin of transient X-ray pulsars and the mechanisms that produce SFXTs are at present unkown. Connections between them would help us to understand common physical properties in transient or suddenly enhanced accretion systems. Thus, future detailed studies of the transient source IGR J01583+6713 would certainly enhance our understanding of the physical origin and connections of both SFXTs and transient X-ray pulsars.

Acknowledgements. We are grateful to the referee for the suggestions of improving the manuscript and to X. L. Zhang for the preparation of some figures. This paper is based on observations of INTEGRAL, an ESA project with instrument and science data center funded by ESA member states (principle investigator countries: Denmark, France, Germany, Italy, Switzerland and Spain), the Czech Republic and Poland, and with participation of Russia and US. W. Wang is supported by the National Natural Science Foundation of China under grants $10803009,10833003$.

\section{References}

Arnaud, K. A. 1996, in Astronomical Data Analysis Software and Systems V, ASP Conf. Ser., 101, 17

Bird, A. J., Malizia, A., Bazzano, A., et al. 2007, ApJS, 170, 175

Corbet, R. H. D. 1986, MNRAS, 220, 1047

Corbet, R. H. D., Markwardt, C. B., \& Tueller, J. 2007, ApJ, 655, 458

Farrell, S. A., Sood, R. K., O’Neill, P. M., \& Dieters, S. 2008, MNRAS, 389, 608

Goldwurm, A., David, P., Foschini, L., et al. 2003, A\&A, 411, L223

Halpern, J. P., \& Tyagi, S. 2005, The Astronomer's Telegram, 681 Ikhsanov, N. R. 2007, MNRAS, 375, 698

Kaur, R., Paul, B., Kumar, B., \& Sagar, R. 2008, MNRAS, 386, 2253

Kennea, J. A., Racusin, J. L., Burrows, D. N., et al. 2005, The Astronomer's Telegram, 673

Kong, A. K. H., Charles, P. A., \& Kuulkers, E. 1998, NewA, 3, 301

Kreykenbohm, I., Wilms, J., Coburn, W., et al., 2004, A\&A, 427, 975

Lebrun, F., Leray, J. P., Lavocat, P., et al. 2003, A\&A, 411, L141

Li, X. D., \& van den Heuvel, E. P. J. 1999, ApJ, 513, L45

Masetti, N., Bassani, L., Bazzano, A., et al. 2006, A\&A, 455, 11

Protassov, R., van Dyk, D. A., Connors, A., Kashyap, V. L., \& Siemiginowska, A. 2002, ApJ, 571, 545

Reig, P., Torrejón, J. M., Negueruela, I., et al. 2009, A\&A, 494, 1073

Schulz, M., \& Mudelsee, M. 2002, Comput. Geosci., 28, 421

Sguera, V., Bazzano, A., Bird, A. J., et al. 2006, ApJ, 646, 452

Steiner, C., Eckert, D., Mowlavi, N., Decourchelle, A., \& Vink, J. 2005, The Astronomer's Telegram, 672

Ubertini, P., Lebrun, F., Di Cocco, G., et al. 2003, A\&A, 411, L131

Wang, W. 2009, MNRAS, 398, 1428 